\title{
ESTIMATION OF THE SHAPE PARAMETER OF GED DISTRIBUTION FOR A SMALL SAMPLE SIZE
}

Prof. Jan Purczyński

Szczecin University

Faculty of Management and Economics of Services

Department of Quantitative Methods

Cukrowa 8, 71-004 Szczecin, Poland

e-mail:jan.purczynski@wzieu.pl

Kamila Bednarz-Okrzyńska, MA

Szczecin University

Faculty of Management and Economics of Services

Department of Quantitative Methods

Cukrowa 8, 71-004 Szczecin, Poland

e-mail: kamila.bednarz@wzieu.pl

Received 28 October 2013, Accepted 1 July 2014

\begin{abstract}
In this paper a new method of estimating the shape parameter of generalized error distribution (GED), called 'approximated moment method', was proposed. The following estimators were considered: the one obtained through the maximum likelihood method (MLM), approximated fast estimator (AFE), and approximated moment method (AMM). The quality of estimator was evaluated on the basis of the value of the relative mean square error. Computer simulations were conducted using random number generators for the following shape parameters: $s=0.5, s=1.0$ (Laplace distribution) $s=2.0$ (Gaussian distribution) and $s=3.0$.
\end{abstract}

Keywords: estimating parameters of GED distribution.

JEL classification: C02, C13, C46. 


\section{Introduction}

In the paper a distribution which is the generalization of Gaussian distribution or Laplace distribution will be considered. The generalized error distribution (GED) includes the specific cases of the Laplace distribution and Gaussian distribution. The density function of which is given by equation (1) is called the Generalized Error Distribution (GED) or Generalized Gaussian Distribution (GGD). Due to a changing value of the shape parameter $s$ (equation 1), the distribution enables modeling of various physical and economic variables. GGD has been applied in image recognition, signal disturbance modeling and speech modeling ${ }^{1}$. Furthermore it is widely applied in image compression, where it is used for modeling the distribution of the discrete cosine transform (DCT) coefficients ${ }^{2}$. GED is successfully applied in modeling the distribution of rates of return for stock indexes and companies ${ }^{3}$. The distributions with the so called "fat tails" have been applied in modeling time-varying conditional variance, among others ${ }^{4}$ and, where for the GARCH model estimation, GED was used as a conditional distribution 5 .

This paper focuses on the problems present in an estimation of the shape parameter $s$ in the case of a small sample size.

We assumed the following symbols for GED density:

$$
f(x)=\frac{\lambda \cdot s}{2 \cdot \Gamma\left(\frac{1}{s}\right)} \cdot \exp \left(-\lambda^{s}|x-\mu|^{s}\right)
$$

where $\Gamma(z)$ - Euler's gamma function.

For $s=1$, GED turns into the Laplace distribution (biexponential):

$$
f(x)=\frac{\lambda}{2} \cdot \exp (-\lambda|x-\mu|)
$$

In the case of $s=2$, a normal distribution is obtained:

$$
f(x)=\frac{\lambda}{\sqrt{\pi}} \cdot \exp \left(-\lambda^{2}(x-\mu)^{2}\right)
$$

where: $\lambda=\frac{1}{\sigma \sqrt{2}}$.

For simplicity reasons, it is assumed that on the basis of the sample, the estimation of parameter $\mu$ was determined:

$$
\widehat{\mu}=\frac{1}{N} \cdot \sum_{k=1}^{N} x_{k}
$$

and consequently, a series of values $x_{k}$ has been centralized by subtracting $\hat{\mu}$. 
Hence the density of the following form is considered:

$$
f(x)=\frac{\lambda \cdot s}{2 \cdot \Gamma\left(\frac{1}{s}\right)} \cdot \exp \left(-|\lambda \cdot x|^{s}\right)
$$

GED characteristics and parameter estimation methods were first mentioned in Subbotin, yet the application of the distribution to statistical issues is owed to Box\&Tiao ${ }^{6}$.

\section{Selected methods of GED parameters estimation}

\subsection{Maximum likelihood method (MLM)}

By applying MLM, the logarithm of likelihood function:

$$
\ln (L(\lambda, s))=N \cdot \ln (\lambda)+N \cdot \ln \left(\frac{s}{2 \Gamma\left(\frac{1}{s}\right)}\right)-\sum_{k=1}^{N}\left|\lambda x_{k}\right|^{s}
$$

from conditions

$$
\frac{\partial \ln (L(\lambda, s)}{\partial \lambda}=0 ; \frac{\partial \ln (L(\lambda, s)}{\partial s}=0
$$

we obtain

$$
\lambda=\left(\frac{N}{s \sum_{k=1}^{N}\left|x_{k}\right|^{s}}\right)^{\frac{1}{s}}
$$

and

$$
g W(s)=s+\Psi\left(\frac{1}{s}\right)+\ln \left(\frac{s}{N} \sum_{k=1}^{N}\left|x_{k}\right|^{s}\right)-\frac{\sum_{k=1}^{N}\left|x_{k}\right|^{s} \ln \left|x_{k}\right|}{\sum_{k=1}^{N}\left|x_{k}\right|^{s}}=0
$$

where: $\Psi(z)=\frac{d}{d z}[\ln \Gamma(z)]$.

From equation (8) the shape parameter $s$ is derived and from equation (7) - parameter $\lambda$. 


\subsection{Approximated fast estimator}

In Krupiński and Purczyński the method of GED parameter estimation based on absolute moments was applied ${ }^{7}$.

An absolute moment of order $m$ is given by:

$$
E_{m}=\int_{-\infty}^{\infty}|x|^{m} \cdot f(x) \cdot d x
$$

From equations (5) and (9) we obtain:

$$
E_{m}=\frac{\Gamma\left(\frac{m+1}{s}\right)}{\lambda^{m} \cdot \Gamma\left(\frac{1}{s}\right)}
$$

Moment estimator $E_{m}$ has a form:

$$
\widehat{E}_{m}=\frac{1}{N} \cdot \sum_{k=1}^{N}\left|x_{k}\right|^{m}
$$

Assuming two different values of moment's $m_{1}$ and $m_{2}$ in equation (10) and eliminating parameter $\lambda$, we obtain:

$$
G(s)=\frac{E_{m_{1}}}{\left(E_{m_{2}}\right)^{\frac{m_{1}}{m_{2}}}}=\frac{\Gamma\left(\frac{m_{1}+1}{s}\right)}{\left[\Gamma\left(\frac{1}{s}\right)\right]^{1-\frac{m_{1}}{m_{2}}}\left[\Gamma\left(\frac{m_{2}+1}{s}\right)\right]^{\frac{m_{1}}{m_{2}}}}
$$

The estimation of the shape parameter $s$ is obtained in the form of an inversion function of the function $G(s)$. The following form of the inversion function was proposed:

$$
\hat{s}=\left(\frac{\ln (G(s)-a}{b}\right)^{\frac{1}{c}}
$$

Table 1 contains values of coefficients $a, b$ and $c$ for particular sets of moment values. The coefficients were derived through computer simulations taking into account the smallest values of the error RRMSE (equation 19), i.e. mean square approximation was conducted, hence the name of the method 'approximated fast estimator' (AFE). 
Table 1. Coefficient values of model (13)

\begin{tabular}{|l|c|c|c|c|}
\hline \multicolumn{1}{|c|}{ Moment values } & $a$ & $b$ & $c$ & Estimate $\hat{s}$ \\
\hline$m_{1}=0.1 ; m_{2}=0.5$ & -0.00925 & -0.016040 & -1.0153 & $\hat{s}_{0}$ \\
\hline$m_{1}=0.5 ; m_{2}=1$ & -0.04606 & -0.074700 & -1.0689 & $\hat{s}_{1}$ \\
\hline$m_{1}=1 ; m_{2}=2$ & -0.12496 & -0.221715 & -1.1374 & $\hat{s}_{2}$ \\
\hline$m_{1}=2 ; m_{2}=3$ & -0.15917 & -0.349350 & -1.1966 & $\hat{s}_{3}$ \\
\hline
\end{tabular}

Source: author's own calculations.

An algorithm proposed in Krupiński and Purczyński is the following ${ }^{8}$. Based on equation (13) and Table 1 , the value of the shape parameter estimate $\hat{s}_{2}$ is determined. Consequently, in accordance with dependency (14), the final estimate $\hat{s} f$ is calculated:

$$
\hat{s} f=\left\{\begin{array}{ccc}
\hat{s}_{3} & \text { for } & \hat{s}_{2}>1.85 \\
\hat{s}_{2} & \text { for } & 1<\hat{s}_{2} \leq 1.85 \\
\hat{s}_{1} & \text { for } & 0.5<\hat{s}_{2} \leq 1 \\
\hat{s}_{0} & \text { for } & \hat{s}_{2} \leq 0.5
\end{array}\right.
$$

Finally, based on the estimate $\hat{s} f$, the parameter estimate $\hat{\lambda}$ is determined:

$$
\lambda=\left[\frac{\Gamma\left(\frac{m+1}{\hat{s} f}\right)}{E_{m} \cdot \Gamma\left(\frac{1}{\hat{s} f}\right)}\right]^{\frac{1}{m}}
$$

where $m=m_{1}$ or $m=m_{2}$.

\subsection{Approximated moment method}

In this paper a modification of the method provided in Krupiński and Purczyński is proposed $^{9}$. The modification is related mainly to the form of the inversion function. By assuming in equation (10) $m_{2}=2 \cdot m_{1}$ and eliminating the parameter $\lambda$, we obtain:

$$
g=\frac{E_{2 m_{1}}}{\left(E_{m_{1}}\right)^{2}}=\frac{\Gamma\left(\frac{2 m_{1}+1}{s}\right) \cdot \Gamma\left(\frac{1}{s}\right)}{\left[\Gamma\left(\frac{m_{1}+1}{s}\right)\right]^{2}}
$$


The inverse function of the function $g$ (equation 16) takes the following form dependent on moment values $m_{2}=2 \cdot m_{1}$ :

$$
\begin{gathered}
m_{1}=0.25 ; m_{2}=0.50 ; \quad \hat{s}_{0}=\left\{\begin{array}{c}
55 g^{-70}+0.73 \text { for } g \leq 1.079 \\
5.7 g^{-28}+0.315 \text { for } 1.079<g<1.132 \\
2.05 g^{-15}+0.18 \text { for } g \geq 1.132
\end{array}\right. \\
m_{1}=0.5 ; m_{2}=1.0 ; \quad \hat{s}_{1}=\left\{\begin{array}{lll}
26 g^{-18}+0.67 & \text { for } & g \leq 1.27 \\
5.5 g^{-9}+0.365 & \text { for } & g>1.27
\end{array}\right. \\
m_{1}=1.0 ; m_{2}=2.0 ; \quad \hat{s}_{2}=\left\{\begin{array}{lll}
29 g^{-7}+0.8 & \text { for } \quad g \leq 2 \\
5 g^{-3}+0.37 & \text { for } \quad g>2
\end{array}\right. \\
m_{1}=2 ; m_{2}=4 ; \quad \hat{s}_{3}=\left\{\begin{array}{l}
12 g^{-1.98}+0.64 \text { for } g \leq 6 \\
6 g^{-1.3}+0.42 \text { for } g>6
\end{array}\right.
\end{gathered}
$$

The final estimate $\hat{s} a$ is obtained from the following dependency:

$$
\hat{s} a=\left\{\begin{array}{c}
\hat{s}_{3} \text { for } \quad \hat{s}_{1}>1.9 \\
\hat{s}_{2} \text { for } 1.4<\hat{s}_{1} \leq 1.9 \\
\hat{s}_{1} \text { for } 0.53<\hat{s}_{1} \leq 1.4 \\
\hat{s}_{0} \text { for } \quad \hat{s}_{1} \leq 0.53
\end{array}\right.
$$

Using equation (15) the estimate of parameter $\hat{\lambda}$ value is obtained.

In order to differentiate from the previously used method - AFE, the proposed method is called 'approximated moment method' - AMM.

\section{Results of computer simulations}

In order to assess the quality of particular estimators, numerical experiments were conducted using a random number GED generator for selected values of the shape parameter: $s=0.5, s=1$ (Laplace distribution) $s=2$ (Gaussian distribution) and $s=3$. The computer simulations consisted in performing $K=2000$ iterations, and on their basis determining the estimation error RRMSE (Relative Root Mean-Squared Error): 


$$
R R M S E=\sqrt{\frac{1}{K} \sum_{k=1}^{K}\left(\frac{\hat{s}_{k}-s_{d}}{s_{d}}\right)^{2}}
$$

where:

$\hat{s}_{k}-$ estimation of parameter $s$ value for $k$-simulation,

$s_{d}-$ exact value of the shape parameter $s$.

In the paper the error RRMSE was determined (equation 19), since it is directly related to the error MSE (Mean-Squared Error):

where: $M S E=\frac{1}{K} \sum_{k=1}^{K}\left(\hat{s}_{k}-s_{d}\right)^{2}$.

$$
R R M S E=\frac{\sqrt{M S E}}{s_{d}}
$$

The mean-squared error is important as such, since it encompasses both the error of the estimator bias and its variance:

$$
M S E=V(\hat{s})+b(\hat{s})^{2}
$$

where:

$V(\hat{s})$ - variance of the estimator,

$b(\hat{s})-$ bias of the estimator.

When presenting the results of computer simulations, the label rmse was used in place of RRMSE.

In order to solve equation (8) the bisection method was applied.

The calculations were conducted for a changing sample size (number of observations) $N=31,41, \ldots, 101$. During the calculations it was noted that the shape parameter error is influenced by the way of determining the mean value, which is subtracted in the process of centralizing. It was especially noticeable in the case of the AFE method, for which the subtracting of the mean value (equation 4) yielded the error rmse several times larger than in the case when the median was subtracted. Therefore in subsequent figures the values of rmse for the samples centralized by means of the median were presented. 
Figure 1 depicts values of error for the generator with the shape parameter $s=0.5$. It should be noticed that the sample with $N=31$ elements has a very large relative error - for ML and AFE the error equals several hundred percent. For other values of $N$ AFE method has the error of $100 \%$.

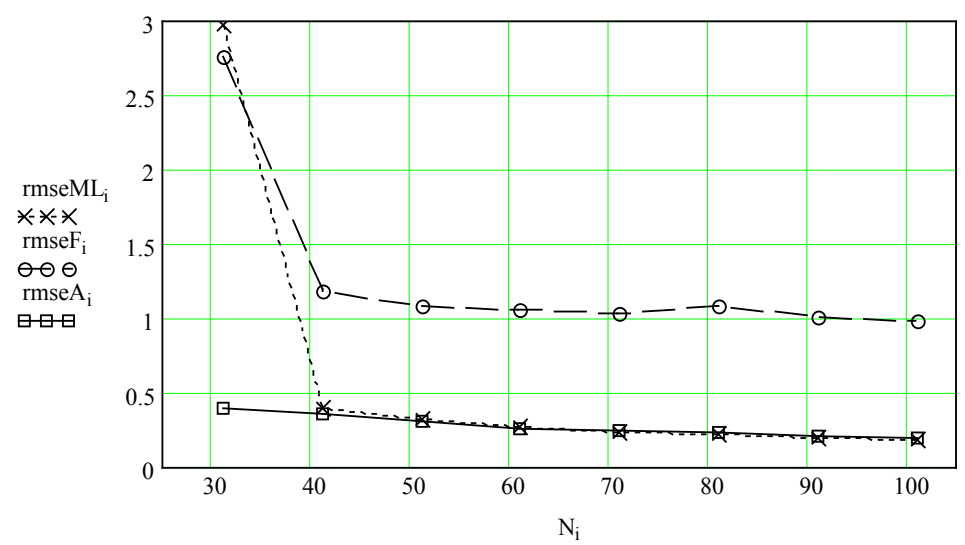

Dotted line with x rmseML presents the values obtained by MLM. Dashed line with circles rmsF corresponds to AFE method (equations 13,14). Solid line with rectangles rmseA marks the values of error obtained through AMM method (equations 17, 18).

Fig. 1. Values rmse obtained for GED generator with the shape parameter $s=0.5$ Source: author's own calculations.

Figures 2 (Laplace distribution) and 3 (Gaussian distribution) show considerable similarity: ML method yields the largest error, and the smallest error - AMM method. Yet the larger the number of $N$ elements, the more similar the errors of particular methods. Figures 1, 2, 3 show the large error of ML for the number of elements $N=31$. Figure $N$ must be substantially large to reveal the advantages of ML method leading to efficient and unbiased estimators. This issue was discussed in Meigen, Meigen ${ }^{10}$. 


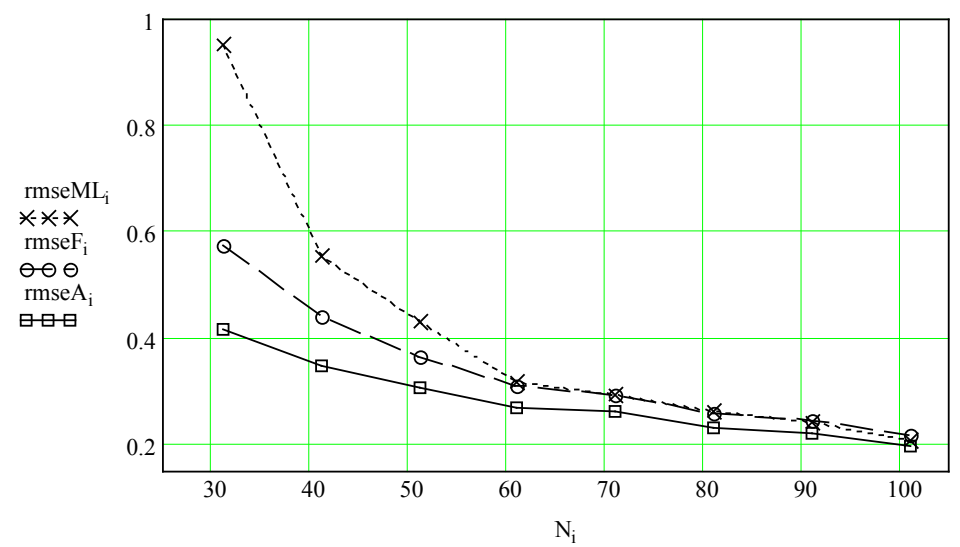

The symbols used in this figure are the same as in Figure 1.

Fig. 2. Values rmse obtained for GED generator with the shape parameter $s=1$ (Laplace distribution)

Source: author's own calculations.

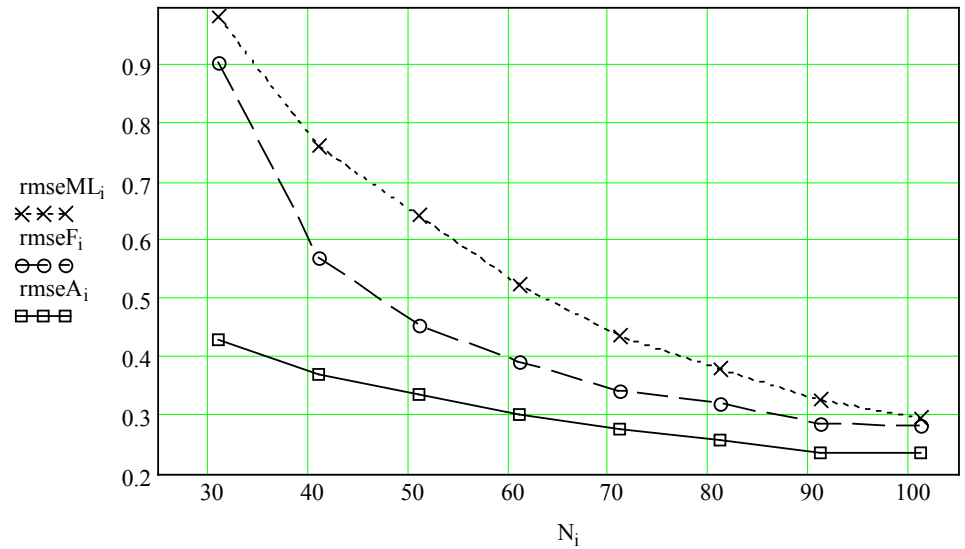

The symbols used in this figure are the same as in Figure 1.

Fig. 3. Values rmse obtained for GED generator with the shape parameter $s=2$ (Gaussian distribution)

Source: author`s own calculations. 


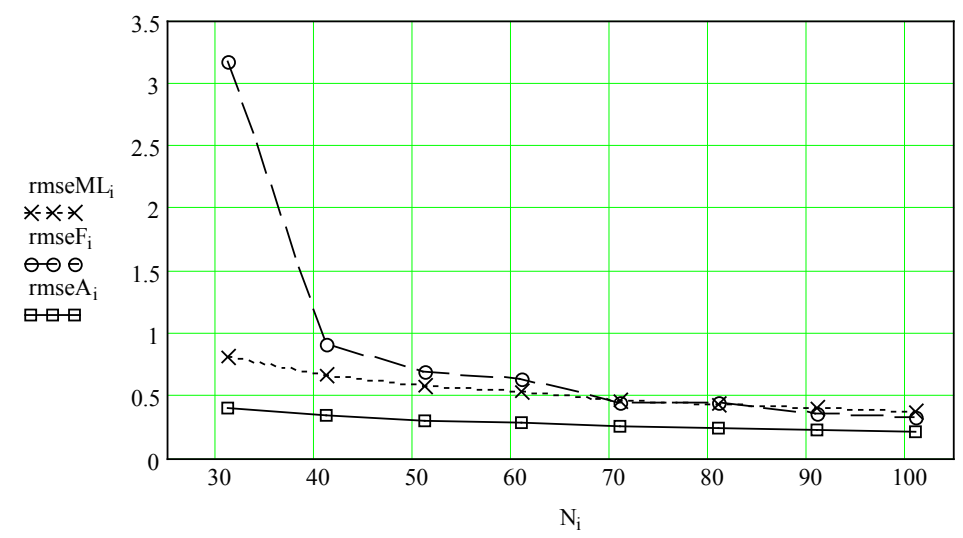

The symbols used in this figure are the same as in Figure 1.

Fig. 4. Values rmse obtained for GED generator with the shape parameter $s=3$

Source: author's own calculations.

In the case of $s=0.5$ and $s=3$ the largest error can be observed for AFE results. The reason for it lies in the form of the function described by equation (13) since it may lead to a complex number, in which case it is advisable to take into account the real part of the derived value. For the generator with $s=0.5$ the complex number appeared in one-fourth of results.

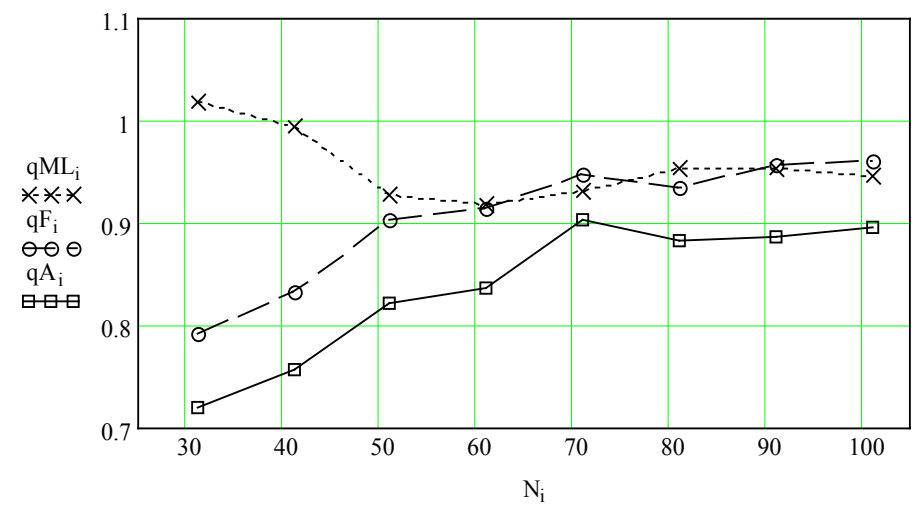

The following curves refer to: MLM- $q M L$ (dotted line with x); AFE- $q F$ (dashed line with circles); AMM- $q A$ (solid line with rectangles).

Fig. 5. Ratio of the value of error rmse yielded by centralizing with the median to the value of rmse for the subtracted mean value - Laplace distribution generator

Source: author's own calculations. 
Figure 5 presents the values of the rmse error obtained by centralizing with the median, divided by the rmse value for the subtracted mean value - Laplace generator. The values smaller than one prove that subtracting the median yields a smaller value of error than subtracting the arithmetic mean. It is particularly visible for the AMM. Coefficient $q M L$ for $s=2$ obtains the value 0.96 and for $s=3 q M L=0.9$. It means that when using ML method, centralizing should be done by subtracting the median. The situation is different for coefficient $q A$, which for $s=2$ slightly exceeds 1 and for $s=3$ is about 1.2. This means that when using the AMM method, the method of centralizing depends on the value of $s$. For $s<1.5$ it is advisable to subtract the median and for $s>1.5$ the arithmetic mean should be used.

\section{Conclusions}

In this paper a new method of estimating the shape parameter s of GED distribution approximated moment method - was proposed. The quality of the proposed estimator was compared with the quality of the estimator obtained through the maximum likelihood method (MLM) and approximated fast estimator (AFE). The quality of estimator was evaluated on the basis of the value of the relative mean square error determined using GED random number generator with the following shape parameters: $s=0.5, s=1$ (Laplace distribution) $s=2$ (Gaussian distribution) and $s=3$.

The method with the smallest error is the one proposed in this paper - the approximated moment method-AMM. As far as other two methods are concerned, for $s=1$ and $s=2$ smaller values of rmse are provided by AFE method. Nevertheless, for $s=0.5$ and $s=3$ MLM is the most accurate.

Attention has been drawn to the fact that the final result depends on whether centralizing is conducted using the arithmetic mean or the median. In the case of MLM, it is advisable to subtract the median. And for AMM, the estimated value of shape parameter $\hat{s}$ should be additionally taken into account. It stems from the fact that for the Laplace distribution, the median is the more efficient estimator than the arithmetic mean estimator. For the normal distribution the situation is reverse and the arithmetic mean estimator is more efficient.

The proposed method is particularly useful for a small sample size $N \leq 100$. For a large sample size, the smallest error is yielded by the estimator obtained by MLM. 


\section{Notes}

${ }^{1}$ Kokkinakis, Nandi (2005), pp. 1825-1858.

${ }^{2}$ Krupiński, Purczyński (2007), pp. 435-441.

${ }^{3}$ Weron, Weron (1998), pp. 284-285.

${ }^{4}$ Hsieh (1989), pp. 339-368.

${ }^{5}$ Nelson (1991), p. 368.

${ }^{6}$ Subbotin (1923); Box, Tiao (1962).

${ }^{7}$ Krupiński, Purczyński (2006).

${ }^{8}$ Ibidem.

${ }^{9}$ Ibidem.

${ }^{10}$ Meigen, Meigen (2006).

\section{References}

Box, G.E.P. \& Tiao, G.C. (1962). A further look at robustness via Bayes theorem. Biometrika, $49(3 / 4)$.

Hsieh, D.A. (1989). Testing for nonlinear dependence in daily foreign exchange rate changes. Journal of Business, 62.

Kokkinakis, K. \& Nandi, A.K. (2005). Exponent parameter estimation for generalized Gaussian probability density functions with application to speech modeling. Signal Processing, 85.

Krupiński, R. \& Purczyński, J. (2006). Approximated fast estimator for the shape parameter of generalized Gaussian distribution. Signal Processing, 86 (4).

Krupiński, R. \& Purczyński, J. (2007). Modeling the distribution of DCT coefficients for JPEG reconstruction. Signal Processing: Image Communication, 22 (5).

Meigen, S. \& Meigen, H. (2006). On the modeling of small sample distributions with generalized Gaussian density in a maximum likelihood framework. IEEE Transactions on Image Processing, 15 (6).

Nelson, D.B. (1991). Conditional heteroskedasticity in asset returns: A new approach. Econometrica, 59 (2).

Subbotin, M.T.H. (1923). On the law of frequency of error. Mathematicheski Sbornik, 31.

Weron, A. \& Weron, R. (1998). Inżynieria finansowa. Warszawa: WNT. 